\title{
Gadget Avalanche: A Technology Literacy Course for Novice Adults
}

\section{Dr. Katy Luchini-Colbry, Michigan State University}

Katy Luchini-Colbry is the Director for Graduate Recruiting at the College of Engineering at Michigan State University, where she completed degrees in political theory and computer science. A recipient of a NSF Graduate Research Fellowship, she received her Ph.D. and M.S.E. in computer science and engineering from the University of Michigan. She has published nearly two dozen peer-reviewed works related to her interests in educational technology and enhancing undergraduate education through handson learning. As a volunteer for Tau Beta Pi, the Engineering Honor Society, Luchini-Colbry facilitates interactive seminars on interpersonal communications and problem solving skills for engineering students across the U.S.

\section{Dr. Dirk Colbry, Michigan State University}




\section{Gadget Avalanche: A Technology Literacy Course for Novice Adults}

\section{Introduction}

Advances in consumer technology have led to a dizzying array of "gadgets" to support everyday tasks. Advertisers promise that the latest smartphones, tablet computers and electronic book readers are easy-to-use and will transform the way that consumers work and play - yet many people find these gadgets to be confusing and frustrating.

We describe the development of a non-credit, personal enrichment course for novice adults that (1) explains the fundamental technologies underlying popular consumer gadgets; (2) guides participants in identifying their goals for using technology; and (3) helps participants find appropriate solutions. The course is designed partly as an opportunity to "touch" new technologies, with the instructors providing a variety of gadgets and participants encouraged to bring their personal devices.

This course was developed as part of the outreach activities of Michigan State University (MSU), and the curriculum was specifically designed to address the needs of adults from the broader community who want to make better use of technology in their personal and professional lives. The course has been offered three times to different groups of adults, with a total audience of approximately 70 adults. This paper presents the curriculum materials in detail, along with lessons learned and suggestions for institutions and instructors desiring to adapt the materials for their own community outreach efforts.

\section{Background}

Mobile devices like smartphones, tablet computers, and e-readers are rapidly changing the way that people work, play and learn. The Pew Research Center's Internet \& American Life Project ${ }^{1}$ has documented dramatic increases in ownership of cell phones, tablet computers and electronic book readers (e-readers) in the last six years (Figure 1). Looking more specifically at internetenabled "smartphones," the Pew Center reported that 45\% of U.S. adults owned a smartphone in $2012 .^{2}$ Google also tracks smartphone ownership in the United States, and reported in $2012^{3}$ that $66 \%$ of U.S. smartphone owners use these devices to access the internet at least once per day; $73 \%$ use their smartphone for email; and 62\% access search engines from their smartphones.

Learning to use a technological "gadget” effectively requires a broad base of knowledge about both the specific task to be accomplished and the underlying technical framework. For example, using a tablet computer to manage email requires knowledge about the task: how to use a particular piece of software to facilitate the process of creating, sending, receiving and managing emails. This task alone can be complex, particularly for novice adults, and it can take time to develop mastery in using electronic mail systems. ${ }^{4}$ In addition to knowledge about the task, users must also develop at least a basic functional understanding of the underlying technology. For instance, how to navigate within the tablet interface (e.g., icons, touch screens, built-in or external keyboards); how to connect the tablet to an appropriate network (e.g., wireless, cellular); and where emails are stored (locally on the device, online in "the cloud," or a combination). 


\section{Adult gadget ownership over time (2006-2012)}

\section{$\%$ of American adults ages $18+$ who own each device}

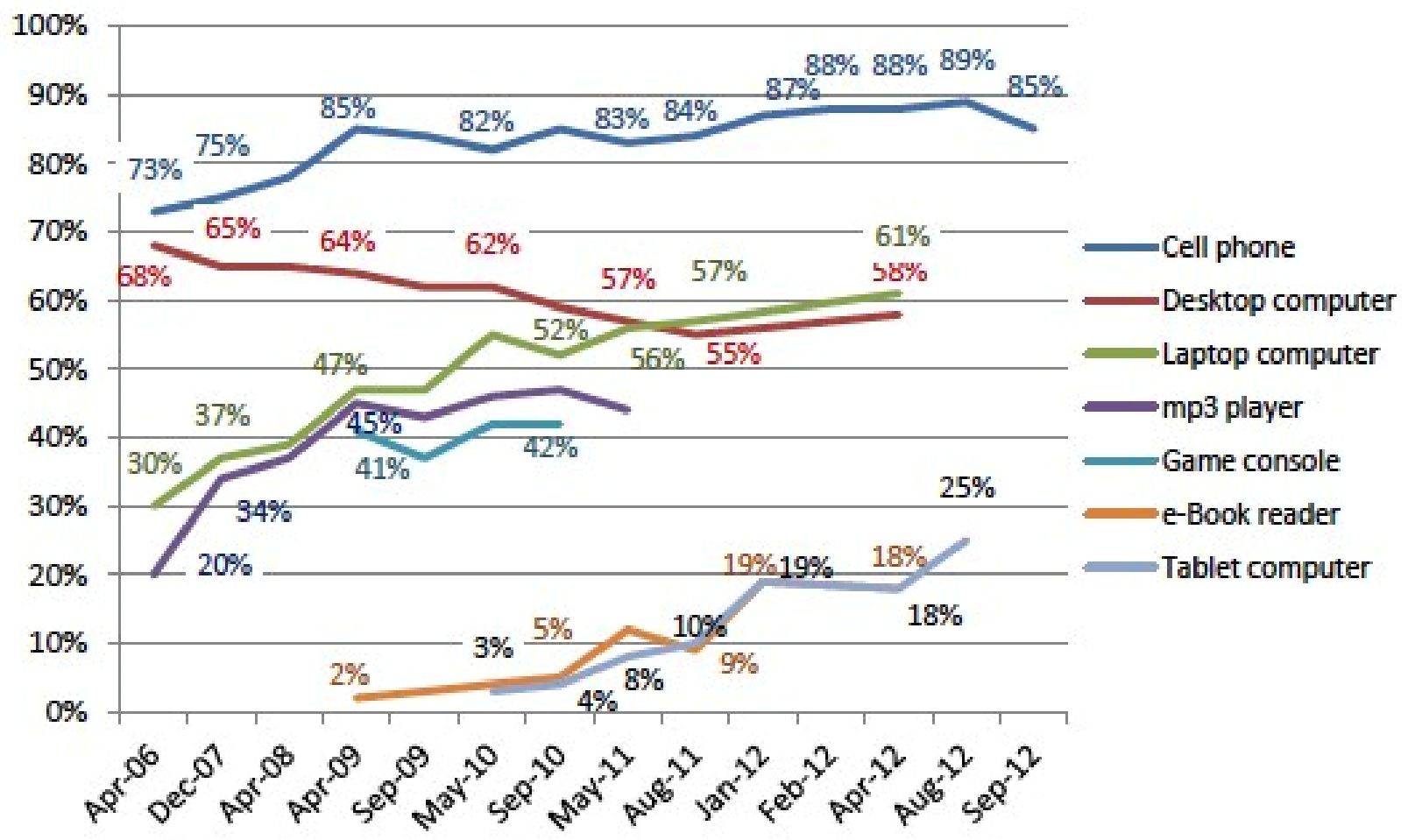

Source: Pew Internet surveys, 2006-2012

Figure 1

\section{Course Structure and Goals}

The "Gadget Avalanche” course was offered three times during spring 2011 and fall 2012; twice as a non-credit personal development course through Michigan State University's "Evening College" community outreach programs (which involve a modest course fee), and once as a free seminar hosted by a unit within the University. All three offerings were open to the general public, and approximately 75 individuals participated in total. Although no demographic information was collected, participants were asked to introduce themselves; from these conversations, we know that at about half of the participants were at or near retirement. Participants were also asked to share their primary goals or reasons for taking the course; common themes included the desire to maintain contact with friends and family; the need to improve technology skills in the workplace; and the goal of learning to use a "gadget" that the individual already owned. The instructors for this course were two University staff members with doctorates in computer science and experience in the design and evaluation of curriculum; one instructor has a background in educational- and learner-centered design, and the other instructor is a specialist in advanced computational hardware. Combined, the instructors have more than two decades of experience developing and delivering personal-development and outreach programs to a range of audiences. 
The initial course was structured in three parts: two, 1-hour classroom sessions spaced one week apart, followed by a 1-hour weekend "field trip" to the local Best Buy technology store to examine the wide variety of available gadgets. During the initial classroom sessions, the instructors provided an overview of key technical concepts, discussed the functionality and features of various mobile computing gadgets, and provided a number of gadgets for participants to try. Many participants brought their personal devices as well, and the classroom sessions were very interactive, with opportunities for questions and for participants to practice various skills inclass. The course-ending field trip to Best Buy was optional, and only four individuals chose to participate. The goal of this trip was to provide access to a far wider range of technology devices than could be provided for in-class experimentation, and the instructors were able to answer questions and suggest specific technologies for those participants who attended. Conversational feedback from the participants revealed part of the reason for the low participation in the field trip: many participants came to class already owning a "gadget" and found that they were satisfied with the information they learned through the classroom activities.

In the second iteration, the course was offered as a free, one-hour "brown bag" seminar hosted by the University's Women's Resource Center. Many of the participants were MSU employees, although several members of the general public also attended. Given the time constraints, the course content was condensed to focus just on Apple-branded devices (iPod, iPad, iPhone). Based on feedback from these first two courses, the curriculum was refined and offered a third time as two, 1.5 hour classroom sessions (without a field trip). The first session focused on developing a basic understanding of key technology terminology (e.g., wi-fi, 3G, cloud computing) and discussing the general functionality of smartphones, tablet computers, and ereaders. During the second session, the focus was on "apps” (applications) and how users could complete specific tasks, such as checking email or reading a book, on different devices.

The curriculum detailed in the remainder of this paper is the result of lessons learned during these three initial course offerings. Throughout the process, the goals of this course have remained the same: (1) to explain the fundamental technologies underlying popular consumer gadgets; (2) to guide participants in identifying their personal goals for using technology; and (3) to help participants find appropriate solutions for meeting these goals.

\section{Course Introduction: The Evolving “Gadget Avalanche”}

As an introduction to the instructors and to the concept of "gadgets," the course began with a description of the various mobile devices that one of the instructors had owned in the previous fifteen years. Through a series of slides (condensed into one image in Figure 2), this "evolution" discussed functionality and devices that proved to be familiar to many of the adult participants in this course. For instance, nearly all participants had used pagers or cell phones for basic communications, and many had used devices to take or share photographs. The instructors were able to quickly assess participants' prior experiences based on their questions and responses during the introduction, which helped guide the instructors' delivery of subsequent course content. More importantly, this introduction illustrated the idea that various gadgets could be used to complete a specific task - and that the "best" gadget choice depends entirely on how the device will be used. 


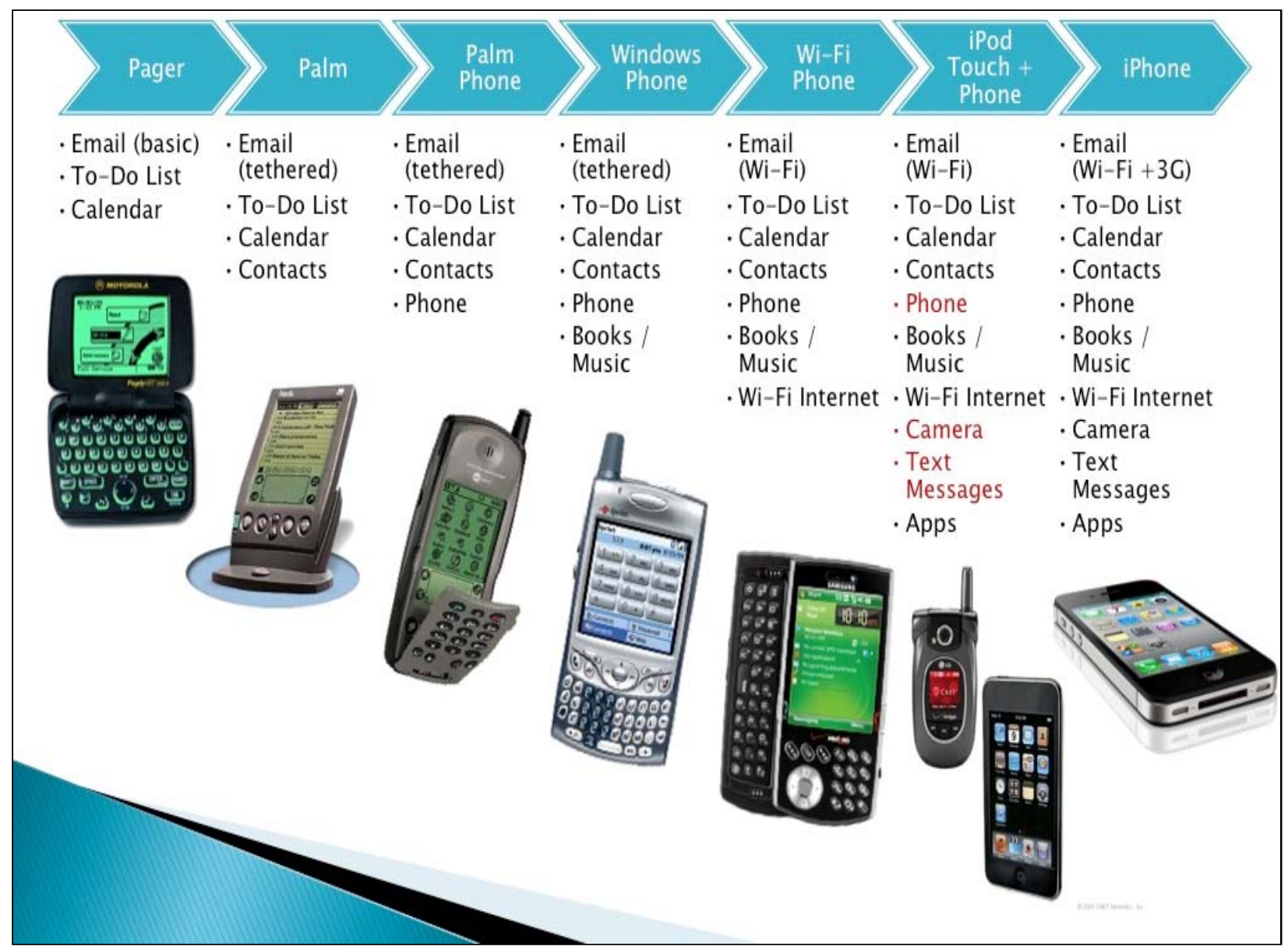

Figure 2: Evolving Functionality of Technology Gadgets

\section{Understanding Network Connections and Cloud Computing}

After the introduction, the focus shifted to explaining some of the basic concepts and technologies that allow gadgets to access information in an "anytime, anywhere" model. The first topic was the difference between connecting devices through "wireless" and "cellular" networks. A basic description was given for key components of each type of network, including common terms such as wireless/Wi-Fi, modem, router, and 3G/4G. Figure 3 shows the graphics used to describe the composition of a wireless network, which typically relies on an access point installed within a home, office or business building. Figure 4 is the graphic used to help describe the broader coverage provided by cellular networks, which offer internet connectivity in most cell phone service areas.

After describing the most common types of network connections, the course discussed the concept of "cloud" computing, or using a network to access, move and store information. The graphic in Figure 5 was used to explain the network of connected computers and devices that create the "cloud," while other examples provided a basic answer to the question "where is the Internet?”. 


\section{Tech Terms: Connections}

- Wireless Network (Wi-Fi, Wireless Internet)

- Your device connects to a wireless access point

- The wireless access point connects to the Internet
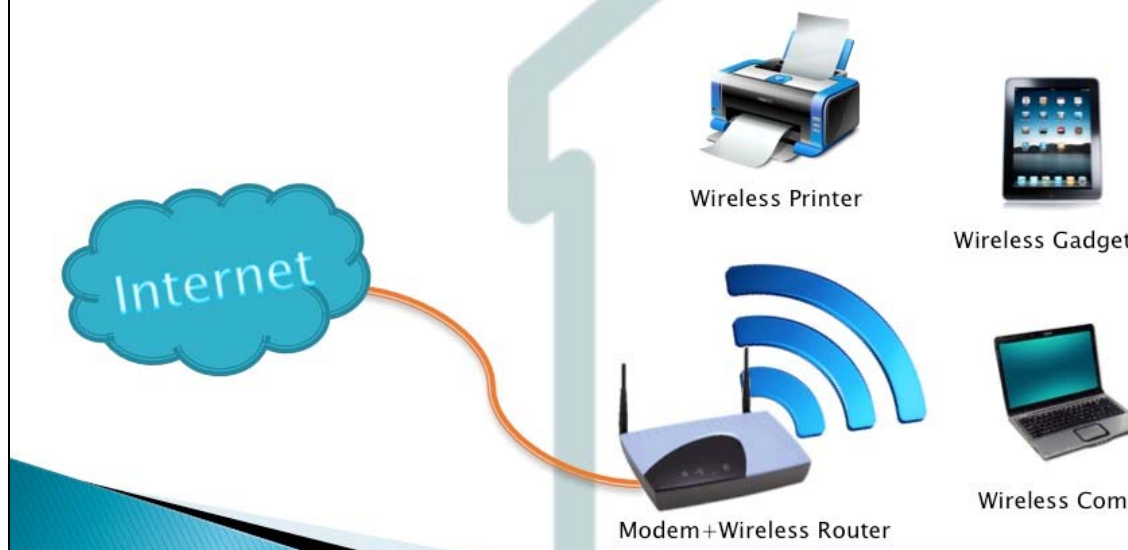

Wireless Gadget

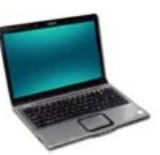

Wireless Computer

Figure 3: Graphical Description of Wireless Network Connection

\section{Tech Terms: Connections}

- Cellular Network (3G, 4G)

Your device connects to a cellular network

The cell network connects to the Internet
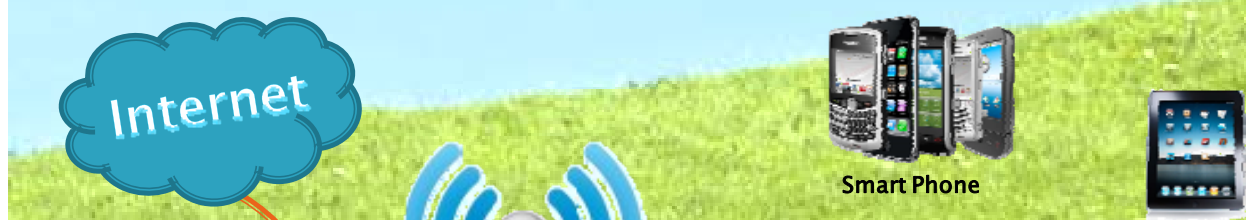

3G Gadget

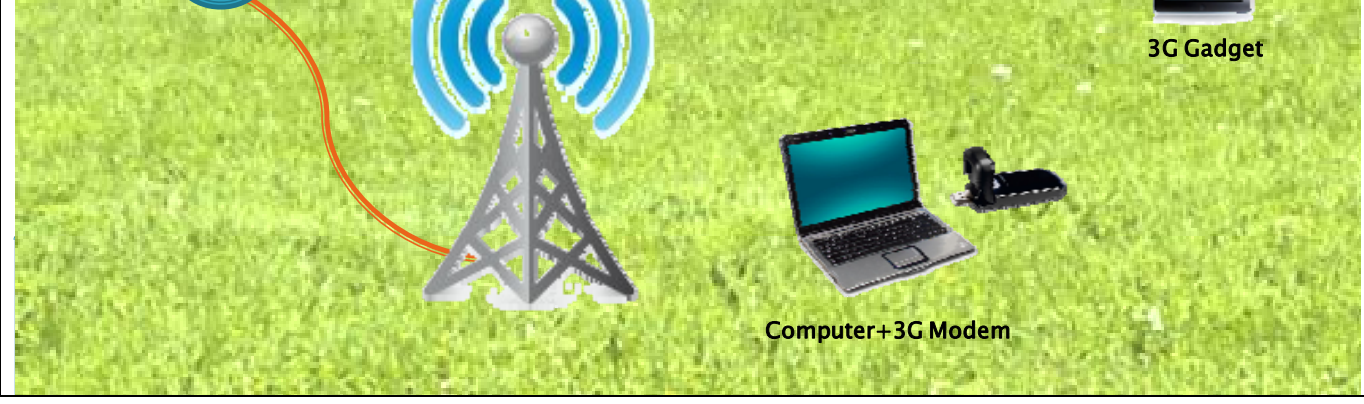

Figure 4: Graphical Depiction of Cellular Network Connection 


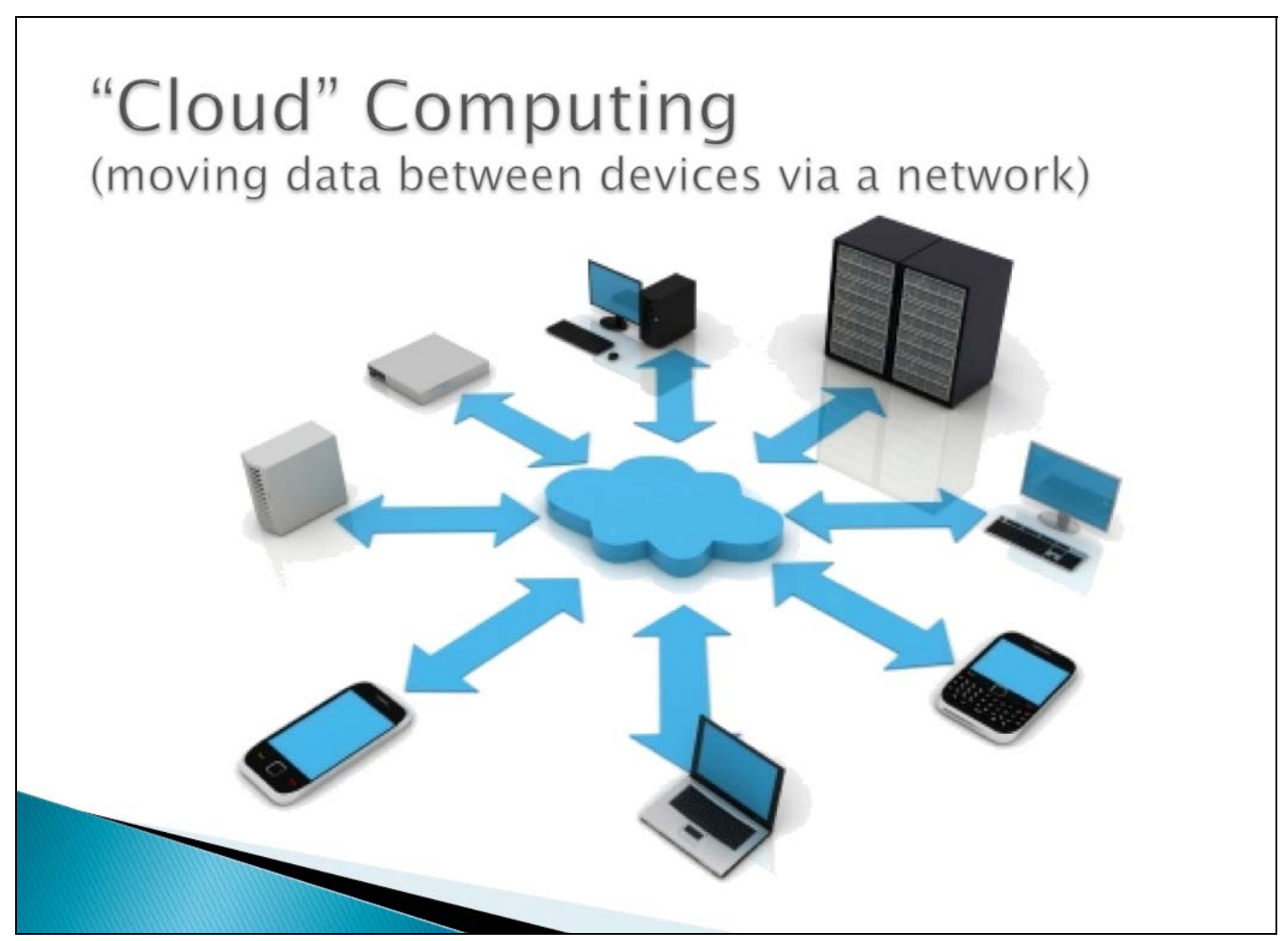

Figure 5: Graphical Description of Cloud Computing

Cloud computing proved to be the most popular topic of conversation - and the greatest source of confusion - for participants in all three classes. A number of concrete examples were used to describe the differences between storing data "in the cloud" and "on a device." For instance, Figure 6 describes an example of purchasing a copy of an electronic book (eBook) through Amazon.com and then accessing that book (from the cloud) on various networked devices.

In contrast, Figure 7 shows the example used to describe the process of storing information locally, in memory on a particular device. In this scenario, the participants were asked to imagine that they were packing for a vacation and, while they were at home, they used their wireless network to download content to their eReader (device for reading electronic books). Since that content is saved and stored locally on the device - rather than in the cloud - they could read their books even when the network connection was turned off ("airplane mode”). Assuming that their eReader also had 3G/4G access, they could access new content during their vacation by turning on the network connection and using a cellular network to download new books from the "cloud." These practical examples proved to be valuable for participants in grasping the concept of cloud computing and different network connection methods. 


\section{Leveraging the Cloud}

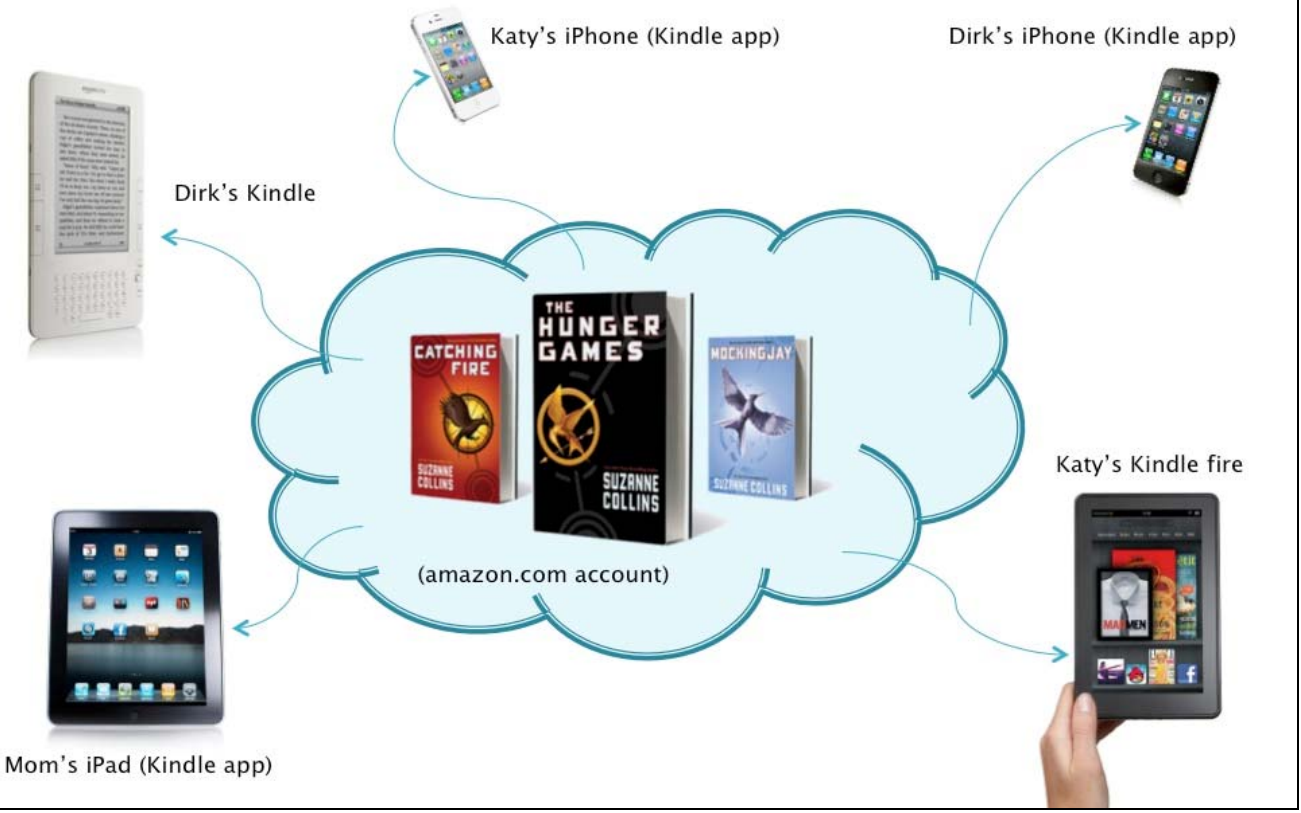

Figure 6: Example of Using Cloud Computing to Access an eBook on Multiple Devices

\section{Leveraging the Device}

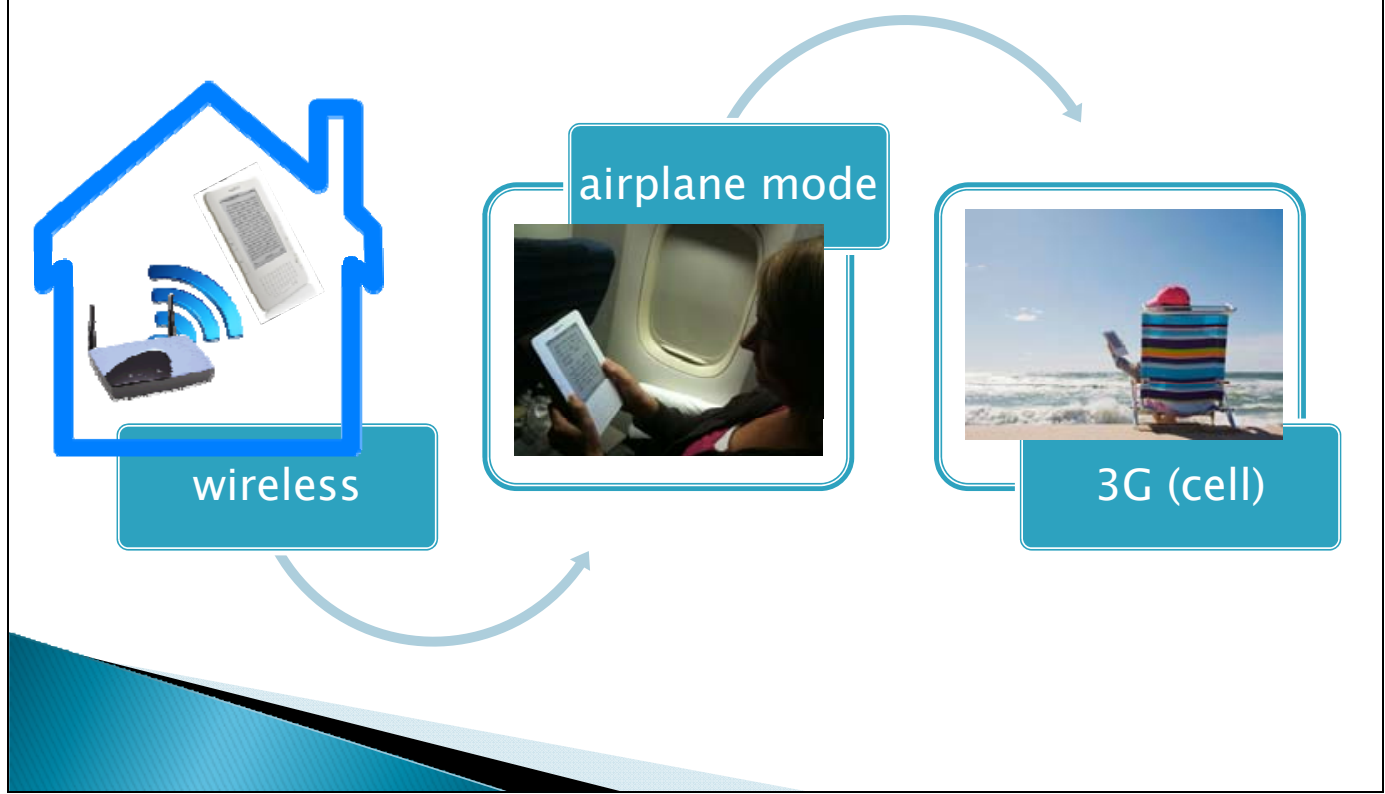

Figure 7: Example of Downloading Content and Storing on a Device 


\section{Goals for Using Gadgets}

Building on the foundational knowledge about functionality, connections and "cloud" computing, the course next explored ways to leverage gadgets to accomplish specific tasks. For this portion of the class, the focus was on using smartphones, tablets and eReaders in professional and personal contexts to accomplish three goals: (1) staying connected; (2) consuming media; and (3) getting organized. Given the diverse audience and the limited timeframe, each goal was introduced in a relatively high-level, functional context and further details were provided only in response to participants' questions. This allowed the instructors to focus attention on those topics of most interest to the participants, while still providing a broad overall framework of knowledge.

Within the context of using gadgets to stay connected, several topics were discussed, including:

- Email, text messages, shared documents

- $\quad$ Phone calls, video calls (Skype, FaceTime, etc.)

- Photos: taking, sharing, printing

- Social Media: facebook, twitter, blogs, etc.

Staying connected with friends, family, and co-workers proved to be a popular topic across all three classes, although the specific focus and questions varied depending on the audience. For instance, in the one-hour seminar attended primarily by MSU employees, little attention was paid to email (which is a common tool for most jobs at the University) while there was a great deal of interest in using social media in both personal and professional settings. The instructors demonstrated FaceTime (a method of making video calls between Apple devices) in all three courses, and discussed the difference between this program (which requires both cellular and wireless internet connectivity) and Skype, which uses wired or wireless network connections to provide similar functionality across a wider range of devices.

Consuming media was another popular topic of conversation for most participants, and the course specifically mentioned the following options:

- Watching: TV / Movies

- Reading: Books, Magazines, Newspapers, websites

- Listening: Audio Books, Music, PodCasts

- Games

Overall, participants' questions about media tended to focus on specific applications (e.g., Netflix, Hulu, KindleReader, Audible) and the pros and cons of specific devices. The final goal - getting organized - was the most pragmatic and generally the one that prompted the fewest questions from participants. The general organizational topics covered were:

- Calendar, To-Do Lists, Contacts

- GPS, Navigation, Maps

- Notes, Documents 
Overall, participants were familiar with these organizational tasks and not all were interested in using a gadget to accomplish them. Again, those questions that did arise regarding organization were generally focused on which applications or tools were most appropriate for specific tasks.

\section{Hardware Options: Comparing Apples to Androids}

Participants had many questions about specific technologies and devices, and the course included a broad overview of the two most common platforms for mobile technologies: Apple (iOS) and Android. The discussion was purposely brief and at a high-level, focusing on some of the key benefits and drawbacks of each platform, as shown in Figure 8a and 8b. The class also discussed overlaps between various devices and the previously discussed goals of staying connected, consuming media, and getting organized, as summarized by Figure 10.

\section{Using Applications to Customize Devices}

The final topic of the class was "apps," or applications that can be installed on various devices in order to accomplish specific tasks. The "app” concept was described in detail, including explanations of why some "apps" work only on certain devices, why some apps are free while others cost money, how to locate available apps, and how to identify "good” apps using recommendation lists and user reviews. The instructors shared some of their personal favorites, and participants shared their own recommendations for apps.

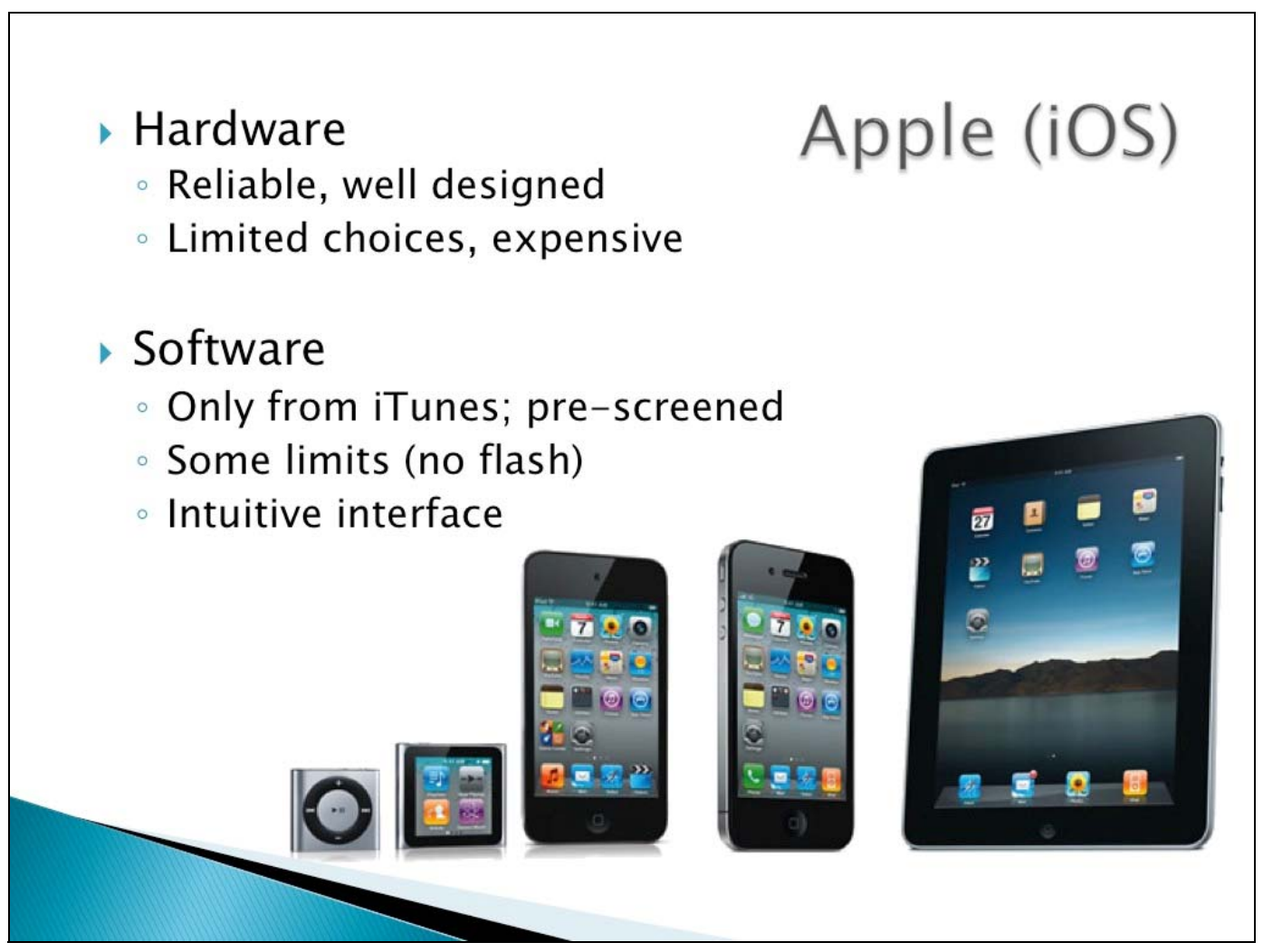

Figure 8a: High Level Comparisons of Apple and Android 


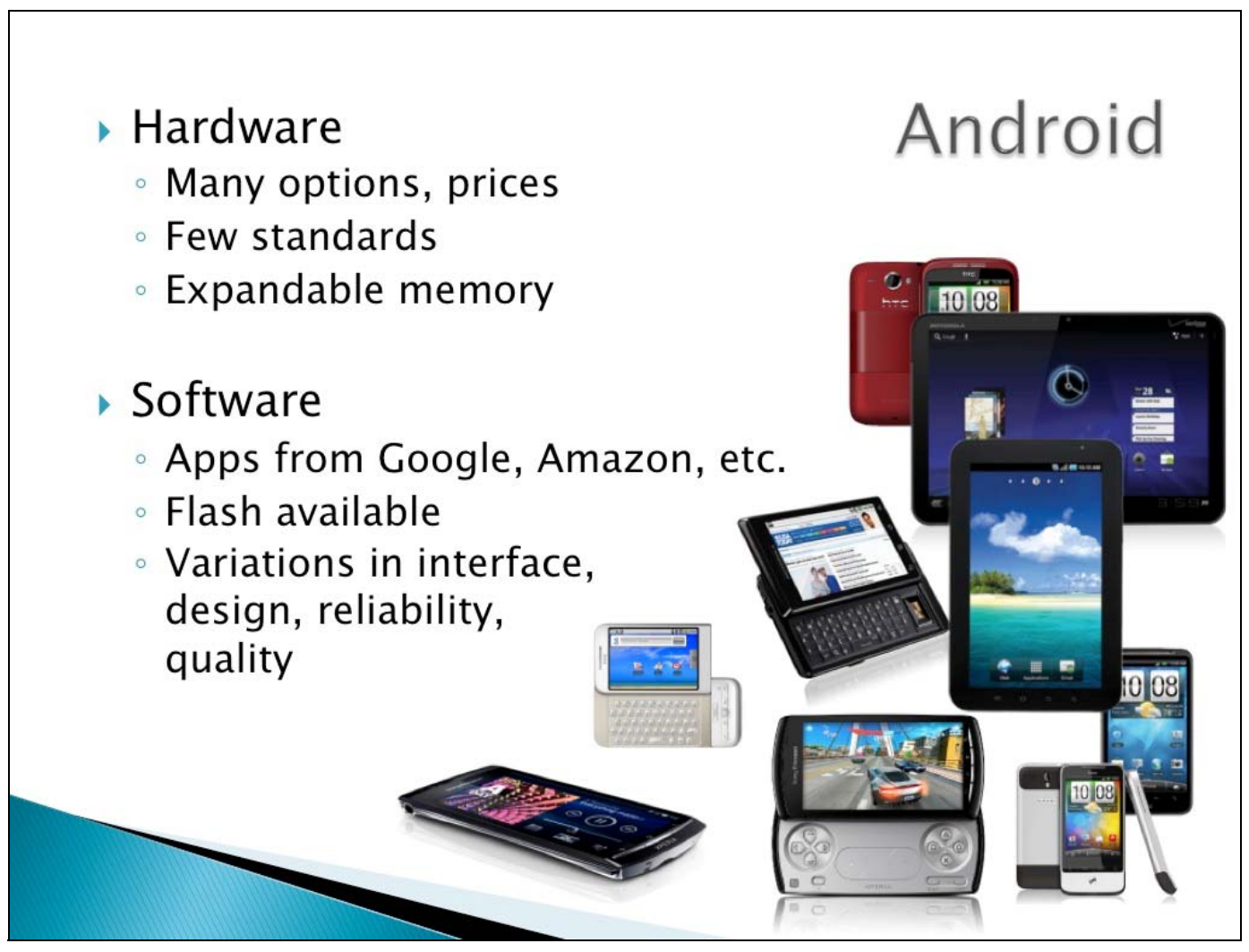

Figure 9b: High Level Comparisons of Apple and Android

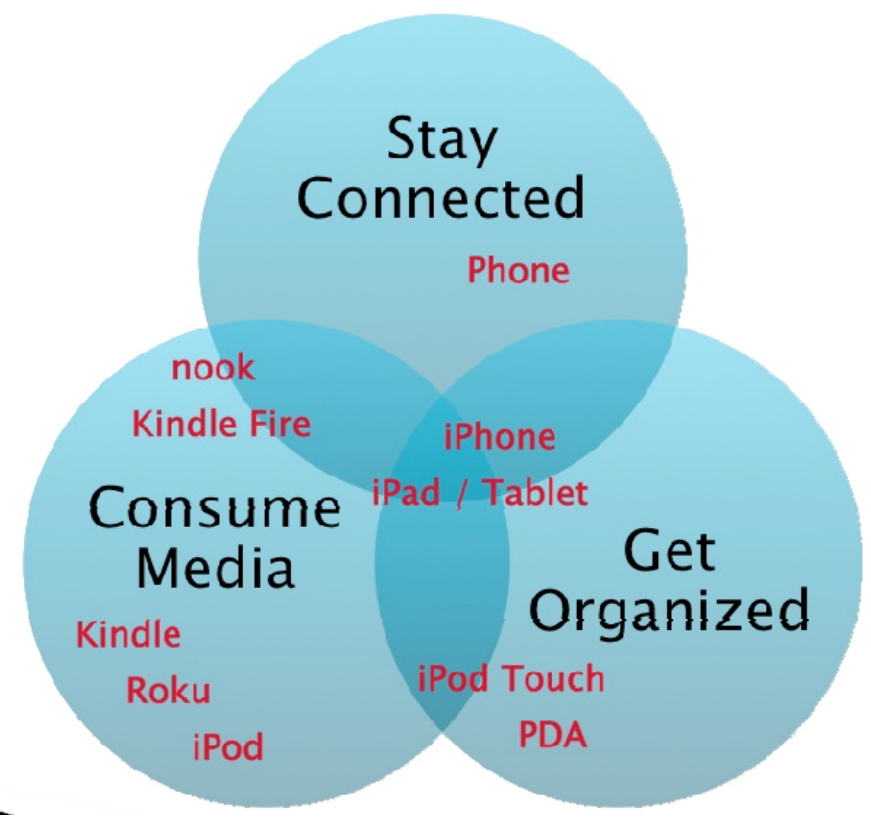

Figure 10: Goals and Devices 


\section{Concluding Discussion}

This paper offers a case study of the development of a non-credit, personal enrichment course for novice adults who want to make better use of technology in their personal and professional lives, or who want guidance in selecting a new gadget to meet specific needs/goals. Although a few specific apps were discussed, the overarching theme of this course was helping participants to identify their goals for using technology, understand which types of devices and connections meet their needs, and knowing how to find applications that can facilitate specific tasks on specific devices.

Participants' feedback proved valuable in evolving and refining the course content over three offerings in 2011 and 2012. As a result, the instructors expanded the explanation of fundamental concepts - such as network connections and cloud computing - and developed several concrete examples that used familiar scenarios to illustrate differences between wireless and cellular connections, or storing information in the cloud or on a device. With this framework in mind, participants were better prepared for subsequent discussions of specific gadgets and of the applications that could be used to make a particular gadget support a particular task.

This curriculum is unique in focusing on novice adult learners, and seeks to provide a balance between getting participants "up to speed" on the use of their own gadgets while also providing foundational information that will allow participants to effectively utilize new gadgets and applications as these technologies continue to evolve. The course has been offered three times to different groups of adults, and has proven to be an effective and popular outreach effort for Michigan State University, with "sold out" classes and requests for additional offerings. The curriculum described here, and the discussion of lessons learned, should prove valuable for institutions and instructors desiring to adapt the materials for their own community outreach efforts.

\section{Acknowledgements}

Funding and administrative support for the courses described here were provided by the Michigan State University Alumni Association and by the MSU Women’s Resource Center.

\section{References}

1. Pew Research Center's Internet \& American Life Project [Internet]. [cited 2013 Jan 5]. Available from: http://pewinternet.org/

2. Smartphone Research: Infographic | Pew Research Center's Internet \& American Life Project [Internet]. [cited 2013 Jan 5]. Available from: http://pewinternet.org/Infographics/2012/Our-Smartphone-Habits.aspx

3. Our Mobile Planet 2012 | Think with Google [Internet]. [cited 2013 Jan 5]. Available from: http://www.thinkwithgoogle.com/insights/featured/our-mobile-planet-2012/

4. Russell AL. Stages in learning new technology: Naive adult email users. Computers \& Education. 1995 Dec;25(4):173-8. 